\title{
Study on Mixed Strategy Nash Equilibrium Based on Rough Set Theory and Particle Swarm Optimization
}

\author{
Cao Li-xia ${ }^{1,2} *$ and Huang Guang-qiu ${ }^{2}$ \\ ${ }^{1}$ College of Science, Xi'an Technological University. Xi'an, 710032, China; ${ }^{2}$ College of Management, Xi'an University \\ of Architecture and Technology. Xi'an, 710055, China
}

\begin{abstract}
There is at least one mixed strategy Nash equilibrium in continuous game, but the existing literature does not provide a universal method of solving infinite strategy mixed strategy NASH equilibrium; this is also the issues to be addressed in the paper. This paper creates a infinitely strategy mixed strategy Nash equilibrium's an approximation algorithm, using rough set theory and the advantages of particle swarm optimization. And proposes the concept of fuzzy game theory, gives a method converted fuzzy game to a classic game theory base on rough set and Vague set theory; The paper provides a theoretical basis for widespread problem in the applications field of game theory when strategy sets and the benefit function problem are fuzzy. The results of the algorithm example show, this paper created algorithm is feasible and effective. It can solve an infinite strategy mixed strategy Nash equilibrium, and equilibrium problem of fuzzy game model.
\end{abstract}

Keywords: Mixed strategy nash equilibrium, particle swarm optimization, rough set, vague set.

\section{INTRODUCTION}

Classic Game theory explains only the meaning of the Nash equilibrium, but doesn't give a general calculation method for the Nash equilibrium [1-3]. As a key of game theory application, many scholars have studied calculation of the Nash equilibrium and computational complexity [4, 5]. These studies results, most of them are unlimited pure strategy Nash equilibrium and limited mixed strategy Nash equilibrium, and the less of them are unlimited mixed strategy Nash equilibrium. Literature [6] gives the existence theorem and an iterative algorithm on unlimited mixed strategy Nash equilibrium, but also only applies to certainty game model, to fuzzy game theory [7], it has a certain limitations. Literature [7] describes three ways change each target's pay matrix $\widetilde{\mathrm{A}}^{\mathrm{k}}$ into clear payoff matrix, and then by selecting the appropriate goal weight change multiple targets countermeasures into a single target countermeasures, or based on Vague linear programming method to solve, but only to study the mixed strategy Nash equilibrium of two-person zero matrix countermeasures. In view of this, the paper systematic study n-person unlimited mixed strategy Nash equilibrium based on rough set theory and methods and particle swarm algorithm, gives the basic idea of the algorithm, finally through examples illustrate the effectiveness of this method.

\section{FOR SOLVING UNLIMITED MIXED STRATEGY GAME NASH EQUILIBRIUM}

Literature [6] points out, when the benefit function is only a limited first-class discontinuity, $\mathrm{n}$ person no cooperative game mixed strategy Nash equilibrium exists; and gives a approximate solution for unlimited mixed strategy Nash equilibrium. This method has certain requirements to the objective function, and thus lacks the breadth of applications. Given the PSO have some advantages and characteristics, such as no continuity requirements to objective function, with fewer parameters than other intelligent optimization, easy coding to achieve, fast convergence, and easy grafting with other intelligent algorithms. In this paper, we give an algorithm with particle swarm algorithm to solve unlimited mixed strategy game Nash equilibrium.

\subsection{The Main idea of the Algorithm}

First, the policy sets of the player $\mathrm{i}$ is divided into $\mathrm{m}$ intervals, if there is discontinuity, the discontinuity points as the cut-off point when divided. The Nash equilibrium solution on the $\mathrm{j}$-th interval is the solution of optimization problems (1):

$\left\{\begin{array}{c}\operatorname{maxF}_{1 j}(X), \max F_{2 j}(X), \ldots, \operatorname{maxF}_{n j}(X) \\ \text { s.t } X_{i} \in \Delta_{i j} i=1,2, \ldots \ldots, n ; j=1,2, \ldots \ldots, m\end{array}\right.$

(1)

Then using PSO algorithm to solve optimization problems (1) on each sub-interval, the solutions are the subsection on pure strategy Nash equilibrium.

Among the particle swarm algorithm, a particle representative the hybrid situation in each game, they search for the optimal position within the space of mixed strategy combinations. The Nash equilibrium is, game strategies are participant's optimal reactions to other policies, thus the particle that represents the Nash equilibrium has the best fitness. In the iterative algorithm, the particles will learn to the individual optimal solution and perform better companion study of 
Table 1. A mixed strategy Nash equilibrium of player i.

\begin{tabular}{|c|c|c|c|c|}
\hline $\mathrm{X}_{\mathrm{i}}$ & $\mathrm{x}_{\mathrm{i} 1}^{*}$ & $\mathrm{x}_{\mathrm{i} 2}^{*}$ & $\ldots \ldots$. & $\mathrm{x}_{\mathrm{im}}^{*}$ \\
\hline \hline $\mathrm{P}_{\mathrm{i}}$ & $\frac{\mathrm{f}_{\mathrm{i} 1}^{*}}{\sum_{\mathrm{j}=1}^{\mathrm{m}} \mathrm{f}_{\mathrm{ij}}^{*}}$ & $\frac{\mathrm{f}_{\mathrm{i} 2}^{*}}{\sum_{\mathrm{j}=1}^{\mathrm{m}} \mathrm{f}_{\mathrm{ij}}^{*}}$ & $\ldots \ldots$ & $\mathrm{f}_{\mathrm{im}}^{*}$ \\
$\sum_{\mathrm{j}=1}^{\mathrm{m}} \mathrm{f}_{\mathrm{ij}}^{*}$ & $\ldots \ldots$ \\
\hline
\end{tabular}

the population based on observation of their game results. Through the study, each player will adjust their strategies, and move in a mixed strategy portfolio space and ultimately tend to the equilibrium point of the game. Finally, using approximate the optimal solution instead of the value of other points on the range, you get an approximation solution of the game mixed strategy Nash equilibrium.

\subsection{Seeking Game Equilibrium Algorithm Step}

Algorithms 1:

1) For a given $\mathrm{j}$, determining the value of the parameter.

Determine the size of the population, the number of particles $\mathrm{H}=20$, learning factor $c_{1}=c_{2}=1.6$; memory update particle number 8 , inertia weight is linear between 0.9 to 0.4 ; iteration termination condition is the maximum number of iterations 50 times; and make evolution algebra $\mathrm{k}=0$.

2) Initialize the position and velocity of all particles (randomly selected).

3) Calculate the fitness value of particles.

Calculate particle and population experienced the best location and update memory. The particles' adapted to the value may be calculated by the objective function. pbest ${ }_{h}^{k}$ Represents the h-th particle evolution $\mathrm{k}$ generation experienced the best position; gbest ${ }^{k}$ represents the entire population evolutionary $\mathrm{k}$ generation experienced the best position. If $\mathrm{k}=0$, then pbest $_{h}^{0}=X_{h}^{0}$, otherwise pbest $_{h}^{k}$ are calculated by the formula (2). gbest $^{k}$ are calculated by the formula (3), and store gbest $^{k}$ into memories. Check whether the iteration termination condition is satisfied, if yes, mark adapted value and the location at this time $\left(\mathrm{X}_{\mathrm{j}}^{*}, \mathrm{f}\left(\mathrm{X}_{\mathrm{j}}^{*}\right)\right)$, go to step (7); If no, go to step (4), among them $X_{j}^{*}=\left(X_{1}, X_{2}, \ldots, X_{n}\right)$ 。

$$
\begin{aligned}
& \text { pbest }_{h}^{k} \\
& =\left\{\begin{array}{cc}
\text { pbest }_{h}^{\mathrm{k}-1} \text { if }: \mathrm{f}\left(\mathrm{X}_{\mathrm{h}}^{\mathrm{k}}\right. & )<f\left(\text { pbest }_{\mathrm{h}}^{\mathrm{k}-1}\right) \\
\mathrm{X}_{\mathrm{h}}^{\mathrm{k}} & \text { otherwise }
\end{array}\right. \\
& \text { gbest }_{\mathrm{h}}^{\mathrm{k}}=\max \left(\text { pbest }_{\mathrm{h}}^{\mathrm{k}}\right) \mathrm{h}=1,2, \ldots \ldots, \mathrm{H}
\end{aligned}
$$

4) With particles of memories library replace those poor fitness in PSO.
Select $\mathbf{J}$ particles from memory library replace those rows after $\mathrm{J}$ bits in the entire swarm.

5) By the formula $\omega=\omega_{\max }-\frac{\omega_{\text {max }}-\omega_{\min }}{\text { iter }_{\max }}$ iter dynamically adjust inertia weight $\omega$.

6) By the formula

$$
\begin{aligned}
& v_{h d}^{k+1}=\varpi v_{h d}^{k}+c_{1} r_{1}^{k}\left(\text { pbest }_{h d}^{k}-x_{h d}^{k}\right)+c_{2} r_{2}^{k}\left(\text { gbest }_{h d}^{k}-x_{h d}^{k}\right) \\
& x_{h d}^{k+1}=x_{h d}^{k}+v_{h d}^{k+1}
\end{aligned}
$$

update velocity and position of the particle, and then go to step (3);

7) When $\mathrm{j}$ take $1,2,3, \cdots, \mathrm{m}$ in turn, repeat steps (1-6), get

Table 1, that is a mixed strategy Nash equilibrium of player $\mathrm{i}$.

Algorithm also can be a viable technology to the initial particles and control the algorithm's iterative steps to ensure that the particles in the iterative process of the algorithm remains in the game mixed strategy

\subsection{Application Examples}

Each particle was represented by mixed strategies of all the players in algorithm, namely: $\mathrm{X}=\left(\mathrm{x}_{1}, \mathrm{x}_{2}, \ldots, \mathrm{x}_{\mathrm{n}}\right)$, according to definition and nature of Nash equilibrium, the fitness function are as follows:

$$
f(x)=\sum_{i=1}^{n} \max \left\{\mu_{i}\left(X \mid s_{j}^{i}\right)-\mu_{i}(X)\right\}, i=1,2, \cdots, n, j=1,2, \cdots, m .
$$

Obviously, by the nature of the Nash equilibrium, if and only if when the mixed situation is Nash equilibrium, the fitness function obtains the minimum value 0 . So the combination of mixed strategies space only fitness of Nash equilibrium point is a minimum.

Offers the player $i=1,2$, policy sets are $S_{i}=[0,1], i=1,2$. We insert two points in policy sets, and then policy sets will be divided into three sub-intervals, benefit function on each interval as shown in Table 2:

Obviously, this strategy is unlimited, and the benefit function has limit number of discontinuities, the mixed strategies Nash equilibrium exists. We use an algorithm 1 to solve, get the game mixed strategies Nash equilibrium $((1,0,0),(1,0,0))$, the results shown in Table 3 :

\section{FUZZY GAME THEORY OF UNLIMITED STRATEGY NASH EQUILIBRIUM SOLVER}

\subsection{The Concept of Fuzzy Game Theory}


Definition: The three elements of the classic game theory, if the policy sets of the player are rough, or benefit function is fuzzy, the rational players should be how to make a 
Table 2. Player's benefit function.

\begin{tabular}{|c|c|c|c|c|}
\hline \multirow{2}{*}{} & \multicolumn{3}{|c|}{ Player 2 } \\
\cline { 2 - 5 } & & {$[\mathbf{0 , 0 . 3 ]}$} & {$[\mathbf{0 . 3 , 0 . 7 ]}$} & {$[\mathbf{0 . 7 , 1 . 0 ]}$} \\
\hline \hline \multirow{3}{*}{ Player 1 } & {$[0,0.3]$} & $(3,3)$ & $(1,0)$ & $(6,1)$ \\
\cline { 2 - 6 } & {$[0.3,0.7]$} & $(0,1)$ & $(0,0)$ & $(4,2)$ \\
\cline { 2 - 6 } & {$[0.7,1.0]$} & $(1,6)$ & $(2,4)$ & $(5,5)$ \\
\hline
\end{tabular}

Table 3. Unlimited strategy game run results with PSO.

\begin{tabular}{|c|c|}
\hline The Number of Calculations & Optimal Fitness \\
\hline \hline 1 & $4.316 \times 10^{-6}$ \\
\hline 2 & $1.424 \times 10^{-6}$ \\
\hline 3 & $3.101 \times 10^{-6}$ \\
\hline 4 & $1.306 \times 10^{-6}$ \\
\hline 5 & $8.700 \times 10^{-7}$ \\
\hline
\end{tabular}

decision-making maximize their own interests' mathematical theory, called fuzzy Game theory.

The fuzzy game theory often were divided into dynamic and static fuzzy game by game repeated number of times; according to the players to understand information about others into complete information and incomplete information fuzzy game. This paper studies only complete information non-cooperative fuzzy static game theory and equilibrium.

Established players of the game, rough set of policies, vague benefits (paid) function constitutes a fuzzy game model. In classical game theory, limited strategy type game exist Nash equilibrium at least, there is at least one mixed strategy Nash equilibrium in continuous game [8]. Here we take the fuzzy model into a classic game model, to find its equilibrium purposes.

\subsection{Solving Fuzzy Game Model}

Rough policy set of the player $i$ is $\left(a_{i}, b_{i}\right)$, it's an equivalence relation $\mathrm{R} \in$ ind $\left(\mathrm{a}_{\mathrm{i}}, \mathrm{b}_{\mathrm{i}}\right)$, we can divide the collection $\left(\mathrm{a}_{\mathrm{i}}\right.$, $\left.b_{i}\right)$ based on $R$ to collection of three classic: $\operatorname{pos}_{R}\left(\left(a_{i}, b_{i}\right)\right)$, $\operatorname{neg}_{R}\left(\left(a_{i}, b_{i}\right)\right), \operatorname{bn}_{R}\left(\left(a_{i}, b_{i}\right)\right)$.

The policy set of player $\mathrm{i}$ binned by continuous attribute discretization method [9].

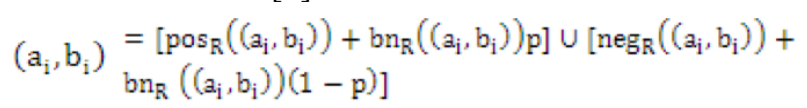

We are located player $\mathrm{i}$ in the bnR $\left(\left(\mathrm{a}_{\mathrm{i}}, \mathrm{b}_{\mathrm{i}}\right)\right)$ of $\mathrm{R}$ with probability p select $\operatorname{pos} R\left(\left(a_{i}, b_{i}\right)\right)$, with probability $1-p$ select negR $\left(\left(\mathrm{a}_{\mathrm{i}}, \mathrm{b}_{\mathrm{i}}\right)\right)$; Then reduction the players' policy set [10], the merger has not resolved the relationship objects; Then with reduction strategies as decision-making conditions, with benefit function as the target condition, the establishment of the decision-making table, resulting in reduction rule combinations, that is exact policy set of the player $i$.
(1) Benefits (paid) function clarity Vague Set of fuzzy benefit function of the player $i$ is

$\left\{<\mathrm{x}, \mathrm{t}_{\mathrm{A}_{\mathrm{i}}}(\mathrm{x}), 1-\mathrm{f}_{\mathrm{A}_{\mathrm{i}}}(\mathrm{x})>\mid \mathrm{x} \in\left(\mathrm{a}_{\mathrm{i}}, \mathrm{b}_{\mathrm{i}}\right)\right\}$, We make fuzzy benefits function clarity by type (5).

$\mathrm{L}\left(\mathrm{E}\left(\mathrm{A}_{\mathrm{i}}\right)\right)=\mathrm{t}_{\mathrm{A}_{\mathrm{i}}}+\mathrm{t}_{\mathrm{A}_{\mathrm{i}}}\left(1-\mathrm{t}_{\mathrm{A}_{\mathrm{i}}}-\mathrm{f}_{\mathrm{A}_{\mathrm{i}}}\right)[7,11]$

If rough policy set of the player $i$ have been precise, fuzzy benefits function have been clarity in $\left[\operatorname{pos}_{R}\left(\left(a_{i}, b_{i}\right)\right)+b_{R}\left(\left(a_{i}, b_{i}\right)\right) p\right]$ and

$$
\left[\operatorname{neg}_{R}\left(\left(a_{i}, b_{i}\right)\right)+b_{R}\left(\left(a_{i}, b_{i}\right)\right)(1-p)\right] \text {, thus, benefit }
$$
function of the player $i$ is average of the right, taking the weighted are $S_{i}^{1}=\frac{\operatorname{Card}\left[\operatorname{pos}_{R}\left(\left(a_{i}, b_{i}\right)\right)\right]+\operatorname{Card}\left[b n_{R}\left(\left(a_{i}, b_{i}\right)\right) p\right]}{\operatorname{Card}\left(a_{i}, b_{i}\right)}$ and

$$
\mathrm{S}_{\mathrm{i}}^{2}=\frac{\operatorname{Card}\left[\operatorname{neg}_{\mathrm{R}}\left(\left(\mathrm{a}_{\mathrm{i}}, \mathrm{b}_{\mathrm{i}}\right)\right)\right]+\operatorname{Card}\left[\mathrm{bn}_{\mathrm{R}}\left(\left(\mathrm{a}_{\mathrm{i}}, \mathrm{b}_{\mathrm{i}}\right)\right)(1-\mathrm{p})\right]}{\operatorname{Card}\left(\mathrm{a}_{\mathrm{i}}, \mathrm{b}_{\mathrm{i}}\right)} .
$$

\section{(2) Solving Fuzzy Game Model}

At this point the model's policy sets are the classic sets, benefits function are deterministic functions, to player $\mathrm{i}(\mathrm{i}=$ $1,2, \ldots, n)$. We can use the classic game theory theories and methods to solve.

\subsection{Algorithm Example}

\subsubsection{Described Problems and Establishing and Models}

We assume there are two online trading platforms in ecommerce transactions; traders can only join a trading platform to conduct e-commerce activities. Thus inter-network trading platform exist game, factors of affecting the platform benefits are many, such as management platform, advertising, popularity index, credibility, profit amount, sales and so on. These factors are game's policy sets among platforms, the platform benefits function is Vague value on a policy set of the player, which formed the two zero and fuzzy game model for the two trading platforms. We use basic idea of this paper to solve:

\subsubsection{Solving the Model}

\section{(1) Policy set precise}

Through the issuance of network questionnaires and statistical analysis, to evaluate management platforms, advertising, popularity index, credibility, profit amount, sales of the importance. the platform the benefits of high, medium, general, low turn assignment is 4,3,2,1, the platform's increase 
Table 4. Players choice of strategies influence platform's income.

\begin{tabular}{|c|c|c|c|c|c|c|}
\hline $\mathrm{Y}_{1}$ & $\mathrm{Y}_{2}$ & $\mathrm{Y}_{3}$ & $\mathrm{Y}_{4}$ & $\mathrm{Y}_{5}$ & $\mathrm{Y}_{6}$ & Income Platform \\
\hline \hline 2 & 4 & 3 & 4 & 1 & 4 & 1 \\
\hline 4 & 3 & 2 & 4 & 3 & 3 & 3 \\
\hline 3 & 1 & 4 & 1 & 4 & 1 & 0 \\
\hline 2 & 4 & 2 & 1 & 1 & 2 & 2 \\
\hline 4 & 2 & 1 & 1 & 1 & 0 \\
\hline 4
\end{tabular}

assigned to 1 , otherwise assigned to 0 , the assignment results are shown in Table 4.

First, the merger has Indiscernible relation objects, thus deleting six and seven two-line in Table 4; then attributes reduction, get reduction attribute set

$$
\left\{\mathrm{Y}_{3}, \mathrm{Y}_{4}\right\},\left\{\mathrm{Y}_{1}, \mathrm{Y}_{5}\right\},\left\{\mathrm{Y}_{1}, \mathrm{Y}_{6}\right\},\left\{\mathrm{Y}_{2}, \mathrm{Y}_{5}\right\}\left\{\mathrm{Y}_{2}, \mathrm{Y}_{6}\right\} \text {, take attribute }
$$
rule $\left\{\mathrm{Y}_{3}, \mathrm{Y}_{4}\right\}$ for data mining, get

$$
\mathrm{Y}_{3 \mathrm{~m}} \mathrm{Y}_{4 \mathrm{~h}} \vee \mathrm{Y}_{3 \mathrm{~g}} \mathrm{Y}_{4 \mathrm{~h}} \rightarrow \text { Platform income " } 1 \text { ". }
$$

That is when the platform's popularity index "medium " or "general" and the credibility "high", the platform will increase benefits. Therefore, precise policies set of the player is $\left\{\mathrm{Y}_{3 \mathrm{~m}}, \mathrm{Y}_{4 \mathrm{~h}}, \quad \mathrm{Y}_{3 \mathrm{~g}}\right\}$.

\section{(2) Benefits function clarity}

According to expert scoring and platform own statistics, we were obtained on weighted average of benefits function, in which, player selects $\mathrm{Y}_{3} \neq, \mathrm{Y}_{4} \bar{*}, \mathrm{Y}_{3}-\mathrm{z}_{\mathrm{R}}, \mathrm{S}_{\mathrm{i}}^{1}, \mathrm{~S}_{\mathrm{i}}^{2}$ are right.

$$
\begin{gathered}
A_{1}=\left(\begin{array}{ccc}
{[0.1,0.2]} & {[0.2,0.4]} & {[0.5,0.7]} \\
{[0.2,0.3]} & {[0.3,0.5]} & {[0.4,0.6]} \\
-[0.3,0.5] & -[0.2,0.6] & {[0.1,0.2]}
\end{array}\right) ; \\
A_{2}=\left(\begin{array}{ccc}
{[0.2,0.3]} & {[0.5,0.6]} & {[0.8,0.9]} \\
{[0.3,0.4]} & {[0.2,0.3]} & {[0.4,0.7]} \\
-[0.3,0.4] & -[0.2,0.3] & {[0.0 .1]}
\end{array}\right)
\end{gathered}
$$

According to formula (5), A_1, A_2 clarity are as follows:

$$
\begin{gathered}
B_{1}=\left(\begin{array}{ccc}
0.11 & 0.24 & 0.60 \\
0.22 & 0.36 & 0.48 \\
-0.36 & -0.28 & 0.11
\end{array}\right), \\
B_{2}=\left(\begin{array}{ccc}
0.22 & 0.55 & 0.88 \\
0.33 & 0.22 & 0.52 \\
-0.33 & -0.22 & 0
\end{array}\right) .
\end{gathered}
$$

Assuming expert scoring and platforms own statistics weights were $0.4,0.6$, two benefits matrix linear assembly, get

$$
B=\left(\begin{array}{ccc}
0.176 & 0.426 & 0.768 \\
0.286 & 0.276 & 0.504 \\
-0.342 & -0.244 & 0.044
\end{array}\right) \text {. }
$$

Based on linear programming, get

$$
\mathrm{x}^{*}=(0.038,0.962,0), \mathrm{y}^{*}=(0.577,0.423,0), \mathrm{v}=0.282 \text {. }
$$

That is, the platform 1 choice popular index "medium" strategy with probability 0.038 , with probability 0.962 choice credibility "high" strategy; platform 2 with probability 0.577 choice popularity index "medium" strategy, with probability 0.423 choice when the credibility of the "high" strategy, the two sides reach a mixed strategy Nash equilibrium, the equilibrium value of 0.282 .

\section{CONCLUSION}

In classical game theory, limited strategy game exist Nash equilibrium at least, there is at least one mixed strategy Nash equilibrium in consecutive game. However, solving mixed strategy Nash equilibrium, most of the existing literature only research limited strategy; relatively studies are few on the unlimited strategy. Using continuous attributes discrete method, this paper divide an unlimited set into number of limit intervals, on each intervals, base on improved particle swarm optimization, give pure strategy Nash equilibrium solution; and then draw unlimited strategy Nash equilibrium of an approximate solution based on the limit theory. The PSO does not ask too much to objective function, convergence is faster, etc., the approximation algorithm has strong adaptability.

Classical game theory does not solve the problem of fuzzy game, but on the current, game theory's application fields, such as politics, economy and culture, there usually appears strategy sets or benefit function fuzzy. Thus, this paper first proposed the concept of fuzzy game theory, gave a way change fuzzy into a classic game theory, which can aid the classic game theory mixed strategy Nash equilibrium balanced approach to solve the fuzzy game theory.

In this paper, the equilibrium solution algorithm and fuzzy game theory research are the development of existing game theories, broadening application scope of this discipline.

\section{CONFLICT OF INTEREST}

The authors confirm that this article content has no conflict of interest.

\section{ACKNOWLEDGEMENTS}

Shaanxi Province Science and Technology Research and Development Program (2013k11-17); 
Xi'an Technological University Fund (XAGDXJJ1324).

\section{REFERENCES}

[1] X. Wang, and Y. Xiao, Game Theory and its Application. Science Press, Beijing, 2008, pp. 15-34.

[2] W. KuhnHarold, Classic Game Theory Han Song, Liu Shijun translated. China Renmin University Press, Beijing, 2005, pp. 817.

[3] W. Liu, and X. Wang, "Review on decision- making in evolutionary games," Operations Research and Management, vol. 17, no. 1, pp. 84-87, 2008.

[4] S. Peng, The Social and Nash Equilibrium Existence Theorems and the Relation of the Theorems. Capital Normal University, China, 2008, pp. 3-5.

[5] N. G. Pavlidis, K. E. ParsoPoulos, and M. N. Vrahatis, "Computing nash equilibrium through computational intelligence methods,"
Journal of Computational and Applied Mathematics, vol. 175, pp. 113-136, 2005.

[6] L. Cao, and G. Huang, "Infinite mixed strategy nash equilibrium's theory and algorithm research," Northwest Normal University (Natural Science edition), vol. 50, no. 3, pp. 14-17, 2014.

[7] X. Zhou, C. Tan, and Q. Zhang, Based on Vague Sets Decision Theory and Methods. Science Press, Beijing, 2008, pp. 156-178.

[8] Y. Li, "Improvement of the response function method in game theory," Statistics and Decision, no. 309, pp. 152-154, 2010.

[9] Ling Wang, "Algorithm of continuous attribute discretization based on improved particle swarm," Computer Engineering and Applications, vol. 49, no. 21, pp. 29-32, 2013.

[10] W. Zhang, W. Wu, J. Liang, and D. Li, Rough Set Theory and Methods. Science Press, Beijing, 2001, pp. 3-10.

[11] W.M. Wang, X. Peng, G. Zhu, J. Hu, and Y. H. Peng, "Dynamic representation of fuzzy knowledge based on fuzzy Petri net and genetic-particle swarm optimization," Expert Systems with Applications, vol. 41, pp. 1369-1376, 2014.

Received: September 16, 2014

Revised: December 23, 2014

Accepted: December 31, 2014

(C) Li-xia and Guang-qiu; Licensee Bentham Open.

This is an open access article licensed under the terms of the Creative Commons Attribution Non-Commercial License (http://creativecommons.org/licenses/by-nc/3.0/) which permits unrestricted, non-commercial use, distribution and reproduction in any medium, provided the work is properly cited. 